\title{
Therapeutic application of RNAi: is mRNA targeting finally ready for prime time?
}

\author{
Dirk Grimm and Mark A. Kay
}

Departments of Pediatrics and Genetics, Stanford University School of Medicine, Stanford, California, USA.

\begin{abstract}
With unprecedented speed, RNA interference (RNAi) has advanced from its basic discovery in lower organisms to becoming a powerful genetic tool and perhaps our single most promising biotherapeutic for a wide array of diseases. Numerous studies document RNAi efficacy in laboratory animals, and the first clinical trials are underway and thus far suggest that RNAi is safe to use in humans. Yet substantial hurdles have also surfaced and must be surmounted before therapeutic RNAi applications can become a standard therapy. Here we review the most critical roadblocks and concerns for clinical RNAi transition, delivery, and safety. We highlight emerging solutions and concurrently discuss novel therapeutic RNAi-based concepts. The current rapid advances create realistic optimism that the establishment of RNAi as a new and potent clinical modality in humans is near.
\end{abstract}

The 2001/2002 discoveries that RNA interference (RNAi) is active in mammals was certainly like a dream come true for researchers and clinicians alike $(1,2)$. For the first time it became possible to effectively silence virtually any gene with a known sequence, either for basic scientific interest or for therapeutic purposes. Particularly appealing is the simplicity with which this can be achieved - theoretically, it is sufficient to exogenously introduce, or alternatively, to intracellularly express, a short double-stranded RNA (dsRNA) with perfect complementarity to a target mRNA. This will result in the incorporation of the active strand of the RNAi trigger (the antisense or guide strand) together with the mRNA into a multiprotein complex known as the RNA-induced silencing complex (RISC). A critical component of RISC, the Argonaute- 2 protein (Ago-2), will then cleave the targeted mRNA at a defined position, followed by its degradation by cellular RNases. Ultimately this leads to the highly efficient and sequence-specific knockdown of this particular gene's expression (3-6) (Figure 1). Not surprisingly, the outstanding potency, simplicity, and specificity of this evolutionarily conserved gene silencing mechanism has fueled a flurry of efforts to develop novel classes of biotherapeutics based on RNAi. Over the past 5 years, a plethora of in vitro and in vivo proof-of-concept studies have showed that practically every human disease with a gain-of-function genetic lesion can become a target for therapeutic RNAi (5, 7-9). These studies have been extensively reviewed in detail in the recent literature, and we refer the reader to these articles for specific diseases [cancer, refs. 10-12; viral infections, refs. 13, 14; neurodegenerative diseases, refs. 15, 16; ocular disorders, ref. 17). Instead, the purpose of the current article is to highlight some of the recently emerging innovative strategies to overcome two general but highly critical hurdles on the way to clinical RNAi translation: delivery and safety. For the first, we will put particular focus on the increasing role of viral-based RNAi vectors, as a potent alternative to small interfering RNA (siRNA) delivery, which is discussed in the accompanying articles in this

Nonstandard abbreviations used: AAV, adeno-associated virus; Ago-2, Argonaute-2; coRNAi, combinatorial RNAi; dsRNA, double-stranded RNA; HBV, hepatitis B virus; hFIX, human factor IX; miRNA, microRNA; RISC, RNA-induced silencing complex; RNAi, RNA interference; shRNA, short hairpin RNA; UTR, untranslated region. Conflict of interest: The authors have declared that no conflict of interest exists. Citation for this article: J. Clin. Invest. 117:3633-3641 (2007). doi:10.1172/JCI34129.
Review Series by Akhtar and Corey $(18,19)$. Subsequently, we will critically discuss the latest and seemingly controversial findings concerning RNAi safety in small animals. In addition, we will review three novel and potentially clinically relevant RNAi-based strategies beyond the silencing of individual target mRNAs.

\section{Toward an ideal vector for in vivo RNAi}

As the RNAi field has rapidly matured since the early reports, it has soon become clear that there will be no universal vector for therapeutic applications in humans. In this respect, the situation with RNAi triggers is no different from previous experiences with other nucleic acid-based biodrugs, in which in vivo delivery posed a major challenge, especially to tissues deep within the body. In fact, there are only a few target tissues of interest that are easily accessible, including the human eye and the respiratory tract. In these two cases, local delivery of siRNAs in saline formulations can result in efficient knockdown of exo- or endogenous targets, i.e., VEGF and its receptor in the eye, or respiratory syncytial virus in the lung $(7,17,20-23)$. Consequently, the first clinical phase I trials evaluating the safety of RNAi in humans are focusing on these particular combinations of tissues and targets (7).

Recent progress with siRNA delivery. Unfortunately, application of "naked" siRNA is not an option for organs or cells deep within the body that are only accessible in a clinically acceptable fashion through systemic RNAi delivery. In these cases, the siRNA not only needs to be protected from serum nucleases, but moreover, efficient and ideally specific delivery to the target tissue becomes more challenging. Therefore, a multitude of academic and industrial groups have recently zoomed in on the pivotal issues of siRNA stability, efficacy, and delivery $(7,24)$. The different strategies and outcomes are discussed in the other Reviews in this Series $(18,19)$. A most notable example of successful systemic in vivo siRNA delivery is a recent report by Zimmermann et al., who demonstrated in some instances, greater than $90 \%$ knockdown of hepatic apoB expression in cynomolgus monkeys following intravenous injection of apoB siRNAs encapsulated in stable nucleic acid lipid particles (SNALPs) (25). Not only was this the first report of efficient systemic RNAi in nonhuman primates, but the silencing effect also lasted for 11 days, which is remarkably long for siRNAs. In fact, the short persistence of siRNA-mediated gene silencing, due to intracellular siRNA degradation, remains another potential hurdle 


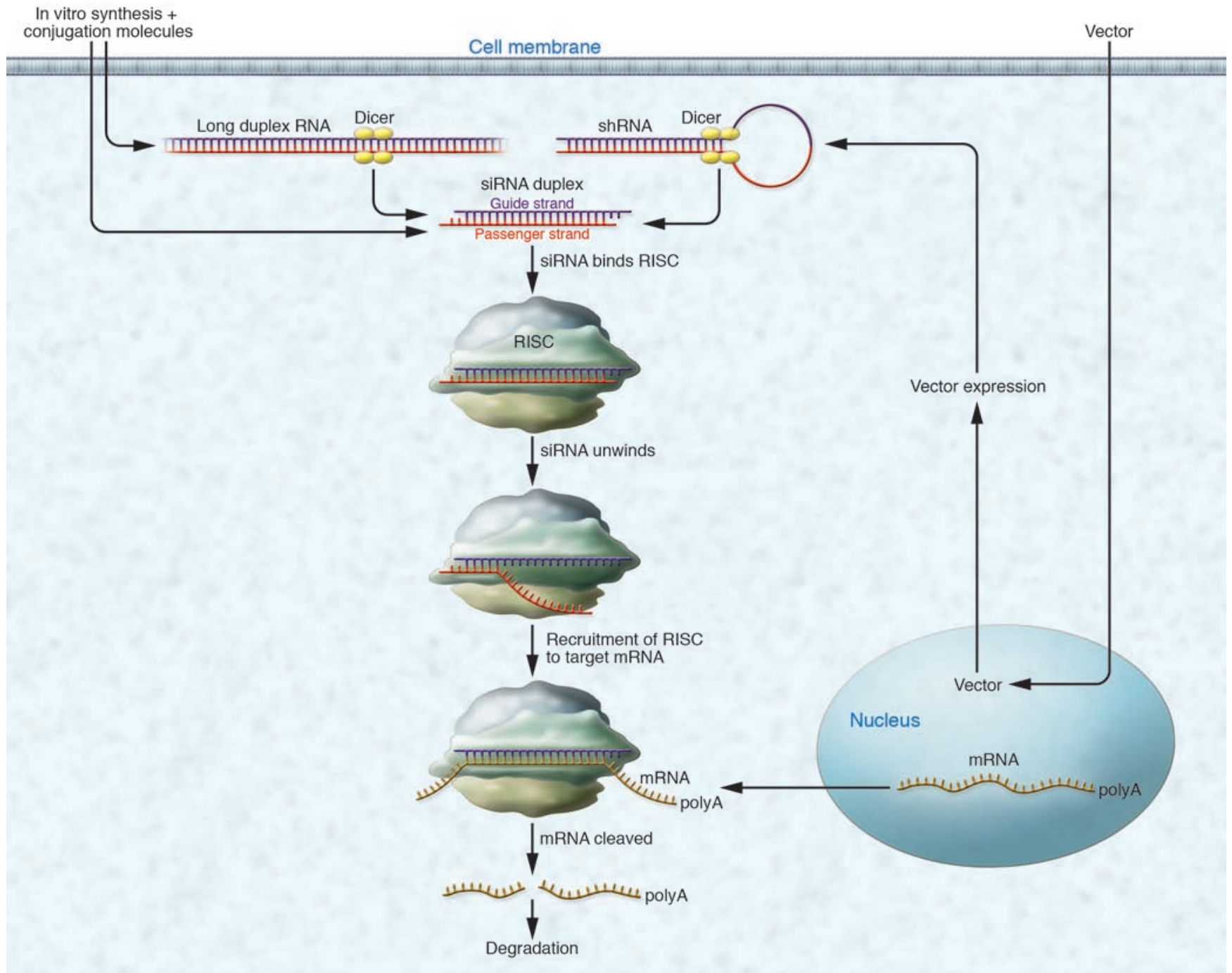

Figure 1

Mechanism of RNAi. dsRNA is cleaved at specific sites by Dicer to form siRNA. siRNA can also be produced either in vitro, after which it can be conjugated to other molecules for efficient delivery into the target cells, or within cells, via DNA-based vectors encoding shRNA. siRNA binds to RISC, the action of which exposes the antisense strand of siRNA and allows it to recognize mRNA with a complementary sequence. Upon mRNA binding to RISC, the mRNA is cleaved and degraded, resulting in the posttranslational silencing of gene expression. Figure modified from ref. 93 .

to their broad clinical application, especially for chronic diseases that require persistent RNAi. One solution might be repeated siRNA delivery, but this will surely conflict with clinical applicability and patient compliance. It is moreover important to note that at the higher doses of SNALP-siRNA conjugates, significant but self-limited hepatocellular toxicity was also reported $(25,26)$. This potential adverse effect will have to be more fully explored prior to clinical implementation.

Nonetheless, there are numerous examples of human diseases in which even short-term suppression of the underlying genes can result in therapeutic benefit and in which siRNAs remain an utmost viable and potent reagent. For brevity, we only wish to point out one intriguing recent study. Kumar et al. presented a chimeric peptide consisting of a 29 amino acid stretch from rabies virus glycoprotein, known to bind the acetylcholine receptor expressed by neuronal cells (27). The authors then added 9 arginine residues to their peptide to mediate binding of siRNAs by charge interaction. Impressively, intravenous injection of the peptide-siRNA complex into mice resulted in transvascular delivery to the brains of the animals. Using an appropriate siRNA, the group could then demonstrate that their approach permits the protection of mice from fatal infection with Japanese encephalitis virus. Indeed, approximately $80 \%$ of the siRNA-treated mice survived for at least 30 days, as compared with $100 \%$ deaths in control groups within 10 days. This study thus strongly exemplifies the enormous potential of judiciously vectorized siRNAs to induce tissue-specific and longlasting therapeutic in vivo RNAi.

Gene delivery vehicles for short hairpin RNA expression. Despite these promising findings and similarly encouraging results with other siRNA delivery systems (e.g., antibody conjugates [ref. 28], aptam- 

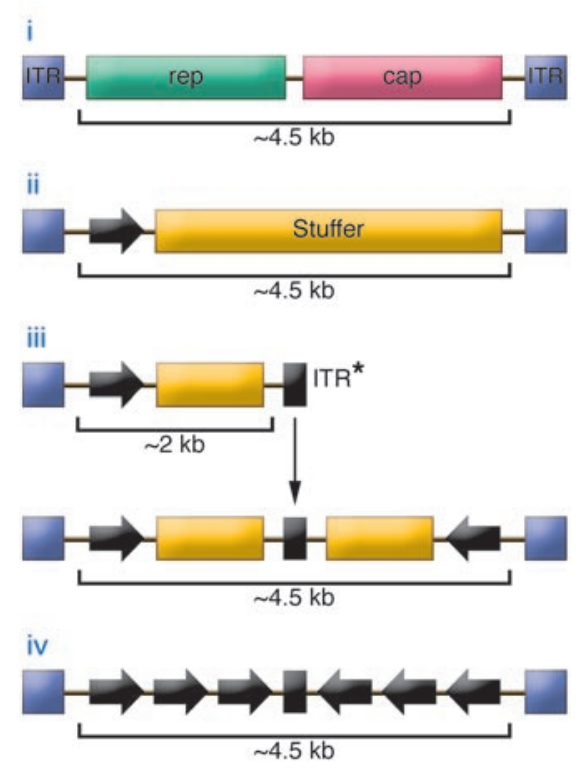

er-siRNA chimeras [ref. 29], and cholesterol-siRNA-lipoprotein complexes [ref. 30]), it is possible that treatment of many human disorders will exceed the capacities of the current siRNA generation. Crucial examples are viral infections and malignancies, both diseases that not only typically affect tissues that are difficult to access with siRNAs but that also require persistent suppression of the underlying gene(s). For the treatment of these challenging classes of diseases, a juxtaposition of RNAi methodology with gene therapy technology appears to be a most promising strategy $(9,14,31)$. The basic concept is to engineer gene delivery vehicles, usually based on a recombinant human or nonhuman virus, for the packaging and transport of an artificial RNAi trigger, typically a short hairpin RNA (shRNA). The latter mimics a naturally occurring precursor microRNA (miRNA) and as such will be processed by the cellular RNAi machinery into an active siRNA $(5,6)$.

The use of viral gene therapy vectors for shRNA administration provides a number of advantages over the delivery of naked or conjugated/complexed siRNAs. First of all, the majority of all available viral vectors have already been assessed clinically in phase I safety trials, with a number also in phase II/III efficacy studies. The gained experience will essentially aid in the design of upcoming vector-based RNAi evaluations. Secondly, a thoughtful choice of viral vector type for a given target will provide the highest possible efficacy and specificity, to a degree beyond that currently achievable with siRNAs. Thirdly, the fact that, in a viral vector, shRNAs are expressed under the control of a promoter opens up a wealth of options for regulating RNAi, including control over tissue distribution and intracellular levels of shRNA expression. Together, the combined options for transductional as well as transcriptional targeting, provided by the viral capsid or the shRNA promoter, respectively, promise a high degree of specificity of in vivo RNAi.

Viral vectors for shRNA delivery. The choice of which viral vector to use for a disease indication is dictated by the inherent properties of the specific vector. Amongst the viral vectors currently under development as RNAi carriers, one of the best candidates are the latest generation of pseudotyped, double-stranded adeno-associated viruses (AAVs) (Figure 2) (31). AAVs are nonpathogenic viruses with an approximately 4.7-kb-long single-stranded DNA genome,

\section{Figure 2}

Classes of AAV vectors for RNAi expression. (i) Wild-type AAV genome. The 2 AAV genes (rep and cap, encoding replication and capsid proteins) are flanked by inverted terminal repeats (ITRs) that serve as replication and packaging signals. (ii) In a conventional single-stranded AAV vector genome, rep and cap are replaced by an shRNA expression cassette. A stuffer sequence is needed to increase the genome size to the packaging optimum. (iii) In an advanced double-stranded AAV vector, the total size of shRNA and stuffer is reduced to less than half the size of wild-type AAV, and 1 ITR is partially deleted (indicated by asterisk). As a result, the vector undergoes a single replication cycle in cells during virus production, leading to duplication of the shRNA/stuffer cassette. In infected cells, the 2 shRNA copies rapidly anneal with each other, which results in instant and robust RNAi expression. (iv) Alternatively, the stuffer DNA can be replaced with further copies of the same (or other) shRNA cassettes to potentiate the RNAi effect or to create a coRNAi vector directed against multiple targets. encapsidated in a non-enveloped, approximately $20-\mathrm{nm}$ protein shell (32). In general, AAVs are highly intriguing as gene therapy vectors, as the wild-type viruses are nonpathogenic in humans and can persistently infect a large variety of dividing and nondividing cell types. Most importantly, AAV typically establishes persistence not by chromosomal integration, like retroviruses, but instead by forming episomal DNA molecules (32). This is critical from a patient safety aspect as it minimizes the risk of insertional mutagenesis from random vector integration, which became apparent as a major drawback of retroviral vectors in a recent clinical trial (33). Also important is that AAV vectors induce no or only minimal $\mathrm{T}$ cell-mediated immune responses in vivo, albeit the outcome might depend on the viral serotype and the targeted tissue (34). Thus far, the most notable result of an anti-AAV immune response in a clinical trial was an asymptomatic transient transaminitis (leakage of liver enzymes without clinical symptoms) $(35,36)$, in striking contrast to the substantially more severe findings with, for example, adenoviral vectors (37). Taken together, AAV vectors are among the safest and thus most promising viral gene delivery vehicles known to date.

With respect to RNAi delivery, AAVs might actually approach our idea of an optimal vector, for several reasons. One is the small inherent size of the viral genome, which is ideally suited for packaging of a single or several shRNA expression cassettes, without the requirement for unwanted "stuffer" sequences (needed in other viral vectors for efficient genome packaging) (Figure 2). Due to the innocuous nature of the AAV particles, high dosing resulting in the transfer of many copies of the therapeutic expression cassette and very high concentrations of siRNA in each cell is easily achieved.

The potential of AAV vectors for RNAi delivery was further significantly increased with two recent advances in the field. In one, vector genomes were engineered for packaging as dsDNA (as opposed to the natural, single-stranded), resulting in particles mediating utmost rapid and robust transgene expression (Figure 2) (38-40). Concurrently, strategies were developed to pseudotype an AAV vector genome with any of the over 100 naturally occurring viral serotypes, many of which are characterized by unique and specific tissue tropisms or other relevant properties (32). Thus 
the use of double-stranded, pseudotyped AAV vectors is a highly promising emerging option for systemic and therapeutic RNAi delivery, and first reports are validating this belief.

For instance, we have engineered double-stranded AAV vectors with an AAV serotype 8 capsid (chosen for its high in vivo efficacy in liver) to express shRNAs against hepatic targets, including hepatitis B virus (HBV) genes and various reporters expressed in mouse livers (38). In a transgenic murine model of persistent HBV infection, systemic delivery of a single low dose of our most robust anti-HBV vector resulted in persistent (at least 5 months) suppression of HBV expression and replication. The outstanding power of this vector system to inhibit $\mathrm{HBV}$ as an example for human liver pathogens was confirmed in a subsequent study by Chen et al., who used another AAV-8-based vector in a similar mouse model (41). Notably, as mentioned, a unique advantage of AAV vectors for RNAi expression is the feasibility to exploit any of the viral capsids (natural or synthetic) for specific tissue targeting. This recently allowed us to silence endogenous genes in the rat retina in vivo, by delivering shRNAs from double-stranded genomes in an AAV serotype 5 capsid (highly efficient in retina) and expressed under an eye-specific promoter (42). Other reported notable examples include the use of AAV serotype 1 for RNAi expression in murine brains in a model of spinocerebellar ataxia (43), or of AAV-2-based vectors for expression of anti-HIV shRNAs (44).

Of course, AAVs are not the only viral vectors under development for RNAi delivery, and further highly promising candidates are currently also emerging from different virus families. These include lentiviral vectors that are based on genetically engineered human immunodeficiency viruses and that show particular potential for RNAi transfer to human embryonic or hematopoietic stem cells $(45,46)$. As such, for instance, a lentiviral RNAi vector was engineered by Rossi and colleagues to express an shRNA directed against HIV (together with other viral inhibitors; see Combinatorial $R N A i$ ), and this vector is currently entering clinical evaluation (5, 47). In this trial, the HIV vector will be used in an ex vivo protocol. Autologous cultured hematopoietic stem cells from HIV patients are incubated with the lentiviral vector. Unlike an AAV vector (which transduces cells without chromosomal integration), the HIV vector will integrate the anti-HIV shRNA expression cassette as a single or at most a few copies into the chromosomal DNA of successfully transduced cells. After bone marrow transplantation, the hope is that the genetically modified transplanted cells will be protected from wild-type HIV, proliferate, and repopulate the bone marrow, eventually replacing the diseased cells. The results of this study are eagerly awaited within the field, as it will be the first clinical evaluation of a vector-based antiviral RNAi strategy.

Finally, a third important example of currently developed viral RNAi vectors are adenoviruses. They might not seem ideal as vectors for shRNA expression because of the strong immunogenicity of the viral capsid, the large size of the viral genome $(\sim 36 \mathrm{~kb}$, requiring inclusion of substantial amounts of stuffer DNA), and reports that some viral genes (virus-associated RNA, which is maintained at least in first-generation adenoviral vectors) can actually inhibit the RNAi pathway (48). Nonetheless, adenoviral vectors are currently emerging as a promising candidate for specific therapeutic RNAi applications, most notably, the treatment of various human cancers (10-12). Especially intriguing are genetic variants of the virus mutated to selectively replicate in and lyse tumor cells. One example was provided by a recent proof-of-concept study by Yoo et al., who engineered an oncolytic adenoviral vector to express
anti-VEGF shRNA (49). Compared with a conventional vector, the novel RNAi construct mediated substantially more robust in vivo antitumor efficacy in glioma xenografts.

Taken together, the recent advances in the field of viral vectors for RNAi expression are clearly highly encouraging, as they include progress on all critical levels: efficacy, tissue specificity, and selectivity. Because this optimization process can largely benefit from our long experience with gene therapy vectors, we anticipate additional and more sophisticated improvements in the near future. Most promising in this regard is a series of recent reports on the genetic engineering of synthetic "designer" AAVs resulting from a directed molecular evolution of wild-type viral capsids. The published approaches include the insertion of cell retargeting peptides into, or PCR-based mutagenesis of, entire capsid genes, or the DNA family shuffling (random mixing of different but related DNA [e.g., capsid genes] sequences) of multiple wild-type viruses (50). All these strategies have the potential to modify and ultimately optimize diverse vector properties, most notably immunogenicity and tissue specificity. This raises hope that we will soon see the first preclinical evaluations of synthetic viral RNAi vectors that can be tailored exquisitely to a specific human disease and cell and tissue type.

\section{Overcoming safety and specificity concerns}

Obviously as with all new therapeutic modalities, the safety of the approach is an essential parameter that requires an utmost thorough preclinical evaluation. In the case of RNAi, the earliest reports of adverse effects date back to 2003, shortly after the first demonstration of RNAi efficacy in human cells, when multiple groups found an induction of IFN responses in siRNA-treated cells $(51,52)$. The initial enthusiasm further waned with additional findings of off-target effects from siRNAs and shRNAs, i.e., the unwanted downregulation of nontargeted mRNAs (53). Subsequent large-scale gene expression profiling studies confirmed that partial sequence homologies were sufficient to induce silencing of off-target genes, in many cases thought to occur through an miRNA-mediated mechanism (i.e., requiring homology of only 6-7 nucleotides [the "seed region"] between the siRNA and the 3 ' untranslated region (3'UTR) of an off-target mRNA] (54-59). Adding to the concerns are recent reports of siRNA-lipid complexinduced stimulation of TLRs, especially TLR7, in plasmacytoid dendritic cells (60). Importantly, it was also found that this effect is cell- and sequence-specific and the result of particular offending GU-rich "danger motifs" in siRNAs, suggesting that this effect can be avoided by using optimized siRNAs and formulations that do not target these cells $(61,62)$. Similarly, multiple reports have shown that chemical modifications of siRNAs can abrogate IFN and cytokine induction $(7,63)$. This further adds to the hope that such side effects can be eliminated altogether in future clinical protocols. Important to note is that the majority of RNAi-associated adverse immune responses have only been observed in cultured cells thus far, and their relevance in vivo remains unclear. Moreover, based on the complexity and differences in the innate responses between animals and humans, further clinical testing of siRNA delivery complexes is required before an accurate assessment of their therapeutic index can be established.

On the other hand, a recent mouse study from our own group has unraveled a novel specific adverse effect of RNAi expression, which had only previously been suggested in in vitro experiments. In this study, we used the above mentioned highly efficient double-stranded AAV-8 vector to express a panel of 49 different 
shRNAs in murine livers (38). At the highest dose, 36 of our vectors caused various levels of liver damage, and nearly half of all tested shRNAs caused animal morbidity and lethality. In line with a previous in vitro report from Bryan Cullen's group (64), we found evidence that high-level shRNA expression had saturated steps of the endogenous miRNA pathway. A key suspect was the nuclear karyopherin exportin-5, which mediates export of both premiRNAs and shRNAs into the cytoplasm (65). Essential from a therapeutic standpoint, we then showed that this in vivo toxicity can be alleviated by the judicious choice of efficient but safe shRNA expression cassettes, and the use of minimal but still highly effective (permitting complete liver transduction) vector doses. Under these circumstances, we were able to potently suppress HBV for 5 months (>2-log drop in viral titers) and various reporter genes for over a year in transgenic mice, clearly demonstrating the potential of the AAV/shRNA approach (38).

Similar nontoxic suppression of hepatic genes was very recently achieved with multiply administered siRNAs in mice and hamsters, although the degree of silencing was not as robust and much more short-lived (less than 4 weeks), typical for siRNAs (66). Based on the saturation model, these findings are not surprising considering that siRNAs enter the RNAi pathway at a late step, presumably circumventing the risk of saturation of upstream components such as exportin-5. However, this latest study is difficult to reconcile with earlier reports showing, for example, that upon coadministration to mouse lungs, two siRNAs can substantially compete with each other and inhibit each other's efficacy in vivo (21). Comparable results were also reported in cultured cells, where the codelivery of multiple shRNAs or siRNAs against various target mRNAs resulted in drops of efficacy of the individual RNAi triggers $(67,68)$. Further strong evidence for the feasibility to saturate the cellular RNAi machinery with high siRNA concentrations came from Hutvagner et al. (69). Their data suggest that cells have a very limited capacity to assemble RISC on exogenously introduced siRNAs and that programmed RISCs can be titrated out in a stoichiometric fashion. This model provides a good explanation for the observed competition between siRNAs; accordingly, two or more siRNAs would compete with each other for a fixed pool of RISC and associated proteins in the cell.

Clearly all these previous results demonstrate the feasibility of also over-saturating RNAi pathways with high doses of siRNAs, at least in certain tissues and cell types. In line with this emerging model, our data in mouse livers indicate that exportin-5 was one but possibly not the only rate-limiting factor in the RNAi pathway (38). Notably, although not yet shown, it is not hard to imagine that another possible limiting protein is the "slicer" component of RISC, Ago-2. Intriguingly, Ago-2 is required by, and binds to, miRNAs, shRNAs, and siRNAs. This could readily explain the repeated observations of siRNA competition and provide a molecular mechanism, i.e., RISC saturation. Support for this model comes from previously published analyses of the expression of Ago proteins in a panel of human tissues. Notably, Ago-2 expression seemed particularly low in liver and lung, the two tissues in which in vivo siRNA or shRNA competition has been implied thus far (70). In fact, this explanation would also be consistent with the recent study by John et al. (66), who mainly used miRNA expression profiles as a readout for potential hepatic toxicity from their high siRNA doses. Adverse saturation of Ago- 2 or other RISC components should affect miRNA function, but probably not their steady-state levels, and thus would have gone unnoticed in miRNA profiles. Also, because only five different siRNAs were tested, more toxic constructs might have simply been missed (66). Most importantly, there are no quantitative data allowing for a reasonable comparison of the intracellular levels of siRNAs and shRNAs in the various models and studies. As shown in our own work, RNAi pathway saturation and thus cytotoxicity are clearly siRNA dose-dependent effects (38); thus it is very likely that discongruent results in recent studies can at least partially be explained by different levels of accumulation of mature siRNAs in the cells.

The sum of these controversial findings highlights the urgent necessity for a better understanding of endogenous RNAi pathways, as a prerequisite to their optimal and safe exploitation. Most critical steps will include, for instance, the characterization and comparison of expression profiles for the different RNAi factors in therapeutically relevant cell types, to unravel whether particular factors are more limiting in certain tissues than in others. It should concurrently be helpful to further unravel the natural interactions of human viruses with the RNAi pathway, based on emerging findings that many viruses actively interact with and often suppress RNAi components in the infected cell $(13,14)$. An intriguing example is adenovirus, which might block endogenous RNAi by swamping and thus saturating exportin-5 with viral RNAs (48). As this is analogous to what might occur in cells expressing high levels of vector-encoded shRNAs, further studies of virus-RNAi interactions might provide us with new mechanisms and concepts to optimize therapeutic RNAi. Likewise, as mentioned, it will be essential to devise means to quantitate and compare mature siRNA levels in vivo, following delivery of different RNAi triggers and using the multiple available vectors.

In the same regard, a most pivotal current effort of many groups is the establishment of novel RNAi promoters based on RNA polymerase II, with the aim to allow regulatable and tissue-specific RNAi expression. Ideally, the use of these promoters will permit the tightly controlled nuclear expression of shRNAs at levels sufficiently high to mediate potent and persistent RNAi while avoiding risks of oversaturating endogenous miRNA pathways, especially exportin-5 and RISC. A variety of such promoter systems have already been adapted from conventional gene expression vectors, including different reversible or permanent variants (reviewed in ref. 71). A potential drawback of this approach, based on the fact that endogenous miRNAs are typically expressed from RNA polymerase II promoters, is that in many cases the hairpin RNAs were embedded in a natural miRNA structure. While this strategy might guarantee the most efficient expression and processing of the hairpin RNA, it might concurrently also increase the risk of saturating additional cellular RNAi components, as these hybrid shRNA/miRNA transcripts will require nuclear cleavage by Drosha (skipped by conventional shRNAs). In general, the ultimate goal toward therapeutic RNAi application is the juxtaposition of these perfected promoter systems with optimized delivery vehicles; obviously this option is restricted to vector-based RNAi expression systems and not available for siRNA-based strategies. The reasonable hope is that this combination will eventually allow for an utmost stringent transductional as well as transcriptional RNAi targeting. Thus by eliminating adverse effects in off-target cells, this will ensure not only highest efficacy but also maximum patient safety (Figure 3).

\section{Novel RNAi-based therapeutic strategies}

Direct targeting of individual genes or mRNAs causally linked to human disease is the most obvious and straightforward clinical 


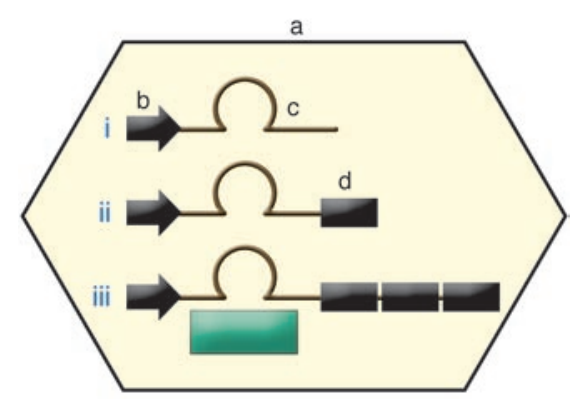

Figure 3

Levels of control over RNAi expression with viral vectors. Through binding to cellular receptors, the viral capsid (a) will determine the tropism of the RNAi vector, i.e., the tissue and cell type that will be infected. This occurs regardless of the vector insert. In a conventional shRNA expression cassette (i), the promoter (b) can further contribute to specificity by being active only in desired tissue or cell types. Alternatively, promoters can be made regulatable via exogenous triggers. Ideally, both properties are combined to permit spatiotemporal control over shRNA expression. Moreover, the shRNA itself (c) is a major determinant of specificity and control and should be designed to selectively bind to the target mRNA. (ii) Theoretically, it should be possible to create hybrid vector genomes in which an shRNA cassette is fused with a binding site for a particular miRNA (d; black box). This would allow the restriction of shRNA expression only to cells in which this miRNA is not expressed, thus helping to minimize off-target effects. (iii) Alternatively, the hybrid genome (or a vector expressing a cDNA; green box) could be fused with multiple tandem sites for miRNA binding and then be used to sequester, and thus inactivate, this miRNA from the cellular pool. This strategy is useful to block miRNAs that are involved in pathogenic processes such as tumorigenesis.

RNAi application, and the literature validating this concept is large and growing exponentially. Yet in recent months, there have also been increasing studies on intriguing and ingenious additional modalities for therapeutic exploitation of RNAi, three of which we will briefly review below.

Combinatorial RNAi. A concern with therapeutic RNAi is that when used to target pathogenic viruses, it might face the same obstacles that have hampered other monotherapies in the past. The biggest challenge is to control viral escape mutants, which can rapidly arise due to the often high viral mutation rate $(13,14)$. This is especially true for RNA viruses, such as hepatitis $\mathrm{C}$ virus (HCV), which can incorporate $10^{-3}$ mutations per viral nucleotide per year, or HIV, which is one of the fastest evolving of all organisms. Unfortunately, in these cases, the superior specificity of RNAi can turn into a disadvantage, as a single nucleotide mismatch between siRNA/shRNA and (viral) target mRNA can abrogate recognition and thus silencing (72). A clever solution to the problem of viral variability is to target their genomes with a cocktail of siRNAs or with vectors expressing multiple shRNAs. Typically these RNAi triggers will simultaneously target different sites within the viral genome and, ideally, sequences that are highly conserved amongst all members of the viral family; the idea is that together these will effectively thwart the formation of escape mutants. Many proof-of-concept studies indeed validate this combinatorial RNAi (coRNAi) approach and suggest that combinations of only three or four siRNAs/shRNAs may already suffice to control even highly mutagenic viruses such as HIV (13). A potent further modification of antiviral coRNAi is to mix viral targets with cellular mRNAs whose products contribute to infection with the pathogen, such as cell receptors or cytoplasmic or nuclear proteins that are being hijacked by the virus.

Importantly, coRNAi strategies also hold promise for the treatment of other human diseases, including cancer. A respective example is the cosuppression of two genes (BRAF and SKP2) that are frequently upregulated and mutated in melanoma cells, resulting in superior antitumor efficacy versus that achieved using a monotherapeutic approach (73). A variant of cancer-specific coRNAi is the synergistic cosuppression of oncogenes and genes that contribute to resistance to chemotherapy or irradiation, in order to enhance the efficacy of conventional cancer drugs. Examples include the P-glycoprotein (encoded by MDR1), whose retrovirus/shRNA-mediated silencing in colon cancer cells resulted in enhanced sensitivity to cytotoxic drugs (74). In another study, adenoviral vector-mediated shRNA expression was used to suppress HIF-1 $\alpha$ (upregulated in rapidly progressing tumors under hypoxic conditions) (75). This not only led to delayed tumor growth in mice, but the effect was further synergistically enhanced by co-irradation, thus validating the concept and power of vectormediated coRNAi gene therapy for cancer treatment.

Yet another modification of coRNAi is to design vectors that express shRNAs in conjunction with either other inhibitors of gene expression, such as ribozymes, or antisense or transdominant proteins (13). A remarkable candidate is the lentiviral vector designed by Rossi and colleagues to combat HIV infection, which is currently entering clinical evaluation. This triple vector coexpresses an anti-HIV shRNA, a ribozyme against the cellular HIV CC chemokine receptor 5 receptor, and an HIV-TAR decoy (to sequester the viral Tat protein) (47). Alternatively, future generations of vectors can coexpress proteins that either serve as reporters or markers or that provide synergistic effects. A first example was a lentivirus coexpressing an shRNA against the human sickle $\beta$-globin, together with a $\gamma$-globin cDNA (76). In differentiating $\mathrm{CD} 34^{+}$cells from sickle cell anemia patients, this vector specifically reduced $\beta^{S}$ while expressing $\gamma$-globin, demonstrating the potential of synergistic gene silencing/addition strategies in the context of stem cell therapies.

Despite these promising initial successes, some further optimization of coRNAi approaches and vectors is still required. A major unknown at this point concerns the genetic stability of viral vectors carrying multiple copies of hairpin RNAs and associated promoters. At least in the context of DNA plasmids, it seems feasible to concatamerize or link together up to 10 shRNA copies, even under a single promoter, while others reported instabilities when hairpin RNA numbers exceeded three (13). Clearly these parameters require more thorough testing and optimization, along with other factors, such as the positioning of multiple hairpins under one or several promoters, and the length and composition of the sequences separating the individual shRNAs. Finally, when using multiple sequences for a given disease target, it will be important to avoid detrimental competition between the individual siRNA incorporation into the RISC (as discussed above), limiting the effectiveness of specific siRNA(s) and defeating the original concept of coRNAi therapy.

miRNAs as therapentic targets. According to current belief, a main function of the RNAi pathway in mammals and humans is the generation and processing of endogenously encoded miRNAs (3-5). Through binding to, and inhibition of, mRNAs with a partially complementary sequence (typically in their $3^{\prime} \mathrm{UTR}$ ), miRNAs 
can posttranscriptionally modulate the expression of probably thousands of genes. This likely occurs mostly through translational inhibition rather than mRNA degradation, although there are clearly overlapping processes, leaving the exact mechanism(s) not well understood $(5,6,77-81)$. This makes miRNAs important regulators of multiple and diverse, benign, or malignant cellular processes such as differentiation, transformation, or viral infection. Not surprisingly, miRNAs therefore offer a vast potential for therapeutic intervention, and several different strategies to study and exploit these molecules have already been explored (82).

In a very exciting recent report, Ebert et al. introduced a novel and clever methodology to specifically and effectively inhibit the function of miRNAs to permit the generation of transient or stable loss-of-function phenotypes (83). In their method, transcripts are expressed from strong polymerase II (e.g., CMV) or III (e.g., U6) promoters that carry multiple (up to 7) tandem binding sites for a given miRNA or an entire miRNA family in their $3^{\prime} U T R$. In transfected cells, these constructs, termed "miRNA sponges," were able to bind and selectively inhibit cognate miRNAs with a complementary heptameric seed. As a result, codelivered reporter genes also carrying binding sites for the same miRNA became potently derepressed. The effect was at least comparable to the best previously reported miRNA inhibitors, including antagomirs (84) and locked nucleic acid (LNA) antisense oligonucleotides (85) when used under standard conditions. Importantly, the sponges also permitted the derepression of endogenous targets that are normally regulated by miRNAs, suggesting a great potential of the approach for target validation. In fact, it was estimated at least in transiently transfected cells, the sponge copy number could reach several thousands per cell, probably enough to inhibit most miRNAs.

From a therapeutic RNAi standpoint, this novel methodology is highly interesting, as it offers a plethora of options to suppress miRNA function using a trigger that is amenable to expression from viral gene therapy vectors (Figure 3 ). Obvious applications would include, for instance, their use to validate miRNA function in tumorigenesis (86) and ultimately to quench cancer-causing miRNAs in animal models and patients. Another intriguing option would be their use to sequester miRNA-122 (miR-122), which is highly expressed in hepatocytes and appears to be required for HCV replication (through binding to the $5^{\prime} \mathrm{UTR}$ ) (87). This could not only offer a novel way to intervene with viral hepatic infection, but moreover, it might be readily combined with other anti-HCV shRNAs and inhibitors as a further example for coRNAi.

Nonetheless, as with all other recently emerging RNAi strategies that are still in their infancy, many parameters of this system need to be better characterized and optimized. For instance, the ideal number of miRNA binding sites and their positioning to each other within the sponge remain unknown, as does the optimal promoter and vector driving their expression. Clearly these and others are all questions that will now be assessed in upcoming animal studies. These looming experiments will also shine more light on the safety and potential risks of this novel methodology. One concern is that near or even full sequestration of endogenous miRNAs could have detrimental effects for the cell, especially given the fact that a single miRNA can probably regulate hundreds or thousands of targets $(80,81)$. This concern would be even greater with sponges that can bind to an entire miRNA family (based on seed specificity). As the function of the vast majority of human miRNAs remains unknown, it cannot be ruled out that concurrent suppression of multiple miRNAs will not be tolerated by the cell and organism.
Segregation of transgene expression. Notably, the above described study by Ebert et al. (83) was not the first to actively exploit binding of endogenous miRNAs to a vector-expressed fusion gene, although the application was unique. In fact, in 2006, Brown et al. had already described their own strategy in which they inserted a binding site for a miRNA (mir-142-3p) into the 3'UTR of the coding sequence for a strong neoantigen, GFP (88). This specific miRNA is selectively expressed in cells of the blood lineage. Here the idea was to systemically deliver the vector to the liver while selectively extinguishing expression from inadvertently transduced blood cells. Indeed, following lentiviral delivery to immunocompetent mice, GFP was only expressed in non-blood cells, particularly in hepatocytes and endothelial cells, but not in hematopoietic lineages. As a result, as GFP expression was absent in APCs, the mice did not mount an anti-GFP immune response, and vector expression was maintained. In a very recent follow-up study (89), the same principle was used to prevent off-target expression of the recombinant clotting human factor IX (hFIX) gene in hematopoietic cells. This thwarted hFIX expression in APCs and thus prevented the elicitation of an anti-hFIX-specific adaptive immune response, which normally hampers long-term hFIX transfer in mice. Indeed, immunocompetent hemophilia B mice treated with the hFIX-mir-142-3p fusion vector showed therapeutic hFIX activity and evidence for phenotypic correction that persisted for over 9 months. Previously, segregation of transgene expression was mainly achieved through transcriptional control, i.e., the use of liver-specific promoters (88). Therefore, the addition of this novel level of RNAi-based posttranscriptional regulation strongly enhances the prospects for vector-mediated gene replacement strategies.

Since Brown et al. (88) used a cross-species transgene in their proof-of-concept study, a scenario not likely to be applied in humans, it will be necessary to confirm the potency of this approach with other transgene and vector combinations. It is particularly tempting and exciting to speculate that these could include shRNA expression cassettes. In fact, a thoughtful choice of miRNA-binding sites fused with shRNA sequences could theoretically result in the segregated and specific expression of RNAi triggers only in the desired target cells, ideally even from systemic RNAi vector administration. Obviously for maximum efficacy and selectivity, this approach could be combined with transductional and transcriptional targeting strategies, i.e., the use of cell- or tissue-specific vectors and promoters (Figure 3 ). On the other hand, the segregation methodology will probably still require the same optimization as the miRNA sponges (83), for instance, the identification of the ideal number and positioning of miRNA-binding sites. Moreover, it is intriguing to note that unlike Ebert et al. (83), Brown et al. did not observe saturation of endogenous mir-142-3p regulation, even when the concentration of their fusion construct reached high copy numbers (88). Notably, Brown et al. had chosen a perfectly complementary target site (88), whereas Ebert et al. had favored typical miRNA-binding sites with a bulge structure in the seed region (83), perhaps explaining this discrepancy. Moreover, differences in cell types and the specific miRNAs could have contributed to the distinct conclusions in both studies. Clearly these and similar novel promising RNAi-based approaches will require intense and thorough further investigation prior to their potential clinical translation.

Also interesting to note in this context of novel RNAi-based expression systems are very recent reports that the design of small dsRNAs against regulatory regions of a gene sequence might shut 
down its expression at the level of transcription (90-92). If such a process does occur in mammalian cells, this approach may be able to turn off a gene by providing even a short and transient burst of the correct dsRNA sequence in the nucleus. This could provide a novel therapeutic approach to control the expression of disease-related genes that, if desired, could again be easily combined with conventional RNAi strategies as well as with the above described miRNA-based systems. Most likely, this new strategy will only be amenable through the use of viral vectors, since at least at this point there are no technologies available that can effectively deliver siRNAs directly into the nucleus.

\section{Conclusions}

An overwhelming plethora of studies conducted to date support the belief that the therapeutic potential of RNAi is nearly unrestricted - theoretically, all annotated disease-related genes should be amenable to RNAi-mediated silencing. Unlike all previously developed similar technologies, RNAi hinges on an endogenous and ubiquitous cellular mechanism, likely explaining its unique and superior efficacy and specificity. Further contrary to all related strategies, RNAi can be induced by multiple variants on the theme of delivering short dsRNAs or hairpin RNAs, from miRNAs to shRNAs and siRNAs, providing researchers and clinicians alike with a variety of options for therapeutic intervention. Additionally, unlike with conventional small-molecule drugs, the same vector platform can probably be used to treat a wide diversity of different diseases within a specific tissue, by merely changing the RNAi sequence within the vector. Classic examples include HBV and $\mathrm{HCV}$, which are very distinct viruses causing different sequelae but, because they infect the same organ (liver), they might be amenable to identical RNAi vector delivery. It is generally indicative of the immense potential of RNAi that translational studies are currently taking place at breathtaking speed, culminating in the first clinical trials already within less than a decade after the basic RNAi discovery in lower organisms.

However, as the field rapidly matures, we are also beginning to realize that routine clinical mRNA targeting might not yet be entirely ready for prime time. Despite the optimism and excite- ment over the first results from clinical evaluations of locally applied siRNAs, it must not be overlooked that new concerns are constantly being unraveled by preclinical studies, and unknown further roadblocks might lie ahead. Therefore, in particular for challenging chronic diseases such as cancer or viral infections, therapeutic RNAi is probably still facing a bumpy road toward proving its worth in looming clinical trials. Most importantly, driving faster on a bumpy road not always makes for a less bumpy ride, and this is especially true for such a potent technology as RNAi. This is clearly implied by the recently accumulating and poorly understood findings of RNAi-associated saturation, competition, and toxicity phenomena in different tissues and in whole animals. Together with earlier reports of adverse immune responses and off-target effects, these data drastically exemplify the paramount necessity to continue to do basic research and to improve our understanding of human RNAi mechanisms to optimize effective application for disease treatment. Fortunately, both the realms of fundamental and translational RNAi research are currently progressing rapidly and synergistically. On one hand, new and detailed insights into the structure and function of endogenous RNAi pathways and their major players are emerging at a lightning pace. Likewise, novel RNAi vectors and applications are being invented and tested at an equally impressive speed, including advanced combinatorial and innovative miRNA-based strategies. All this raises considerable hope that a revolutionary transformation in modern medicine is on the horizon and that an arsenal of effective and safe systemic RNAi therapies for a wide range of human diseases may indeed be realized within a few years.

\section{Acknowledgments}

This work was sponsored by grants from the NIH (AI071068 and DK078424).

Address correspondence to: Mark A. Kay, School of Medicine, Departments of Pediatrics and Genetics, Stanford University, 300 Pasteur Drive Room G305, Stanford, California 94305, USA. Phone: (650) 498-6531; Fax: (650) 498-6540: E-mail: markay@stanford.edu.
1. Elbashir, S.M., et al. 2001. Duplexes of 21-nucleotide RNAs mediate RNA interference in cultured mammalian cells. Nature. 411:494-498.

2. McCaffrey, A.P., et al. 2002. RNA interference in adult mice. Nature. 418:38-39.

3. Dykxhoorn, D.M., and Lieberman, J. 2005. The silent revolution: RNA interference as basic biology, research tool, and therapeutic. Annu. Rev. Med. 56:401-423.

4. Hannon, G.J., and Rossi, J.J. 2004. Unlocking the potential of the human genome with RNA interference. Nature. 431:371-378.

5. Kim, D.H., and Rossi, J.J. 2007. Strategies for silencing human disease using RNA interference. Nat. Rev. Genet. 8:173-184.

6. Tomari, Y., and Zamore, P.D. 2005. Perspective: machines for RNAi. Genes Dev. 19:517-529.

7. Bumcrot, D., Manoharan, M., Koteliansky, V., and Sah, D.W. 2006. RNAi therapeutics: a potential new class of pharmaceutical drugs. Nat. Chem. Biol. 2:711-719.

8. de Fougerolles, A., Vornlocher, H.P., Maraganore, J., and Lieberman, J. 2007. Interfering with disease: a progress report on siRNA-based therapeutics. Nat. Rev. Drug Discov. 6:443-453.

9. Grimm, D., and Kay, M.A. 2006. Therapeutic short hairpin RNA expression in the liver: viral targets and vectors. Gene Ther. 13:563-575.

10. Gartel, A.L., and Kandel, E.S. 2006. RNA interference in cancer. Biomol. Eng. 23:17-34.

11. Pai, S.I., et al. 2006. Prospects of RNA interference therapy for cancer. Gene Ther. 13:464-477.

12. Takeshita, F., and Ochiya, T. 2006. Therapeutic potential of RNA interference against cancer. Cancer Sci. 97:689-696.

13. Grimm, D., and Kay, M.A. 2007. Combinatorial RNAi: a winning strategy for the race against evolving targets? Mol. Ther. 15:878-888.

14. Leonard, J.N., and Schaffer, D.V. 2006. Antiviral RNAi therapy: emerging approaches for hitting a moving target. Gene Ther. 13:532-540.

15. Davidson, B.L., and Boudreau, R.L. 2007. RNA interference: a tool for querying nervous system function and an emerging therapy. Neuron. 53:781-788.

16. Raoul, C., Barker, S.D., and Aebischer, P. 2006. Viral-based modelling and correction of neurodegenerative diseases by RNA interference. Gene Ther. 13:487-495.

17. Campochiaro, P.A. 2006. Potential applications for RNAi to probe pathogenesis and develop new treatments for ocular disorders. Gene Ther. 13:559-562.

18. Akhtar, S., and Benter, I.F. 2007. Nonviral delivery of synthetic siRNAs in vivo. J. Clin. Invest. 117:3623-3632.
19. Corey, D.R. 2007. Chemical modification: the key to clinical application of RNA interference? J. Clin. Invest. 117:3615-3622.

20. Shen, J., et al. 2006. Suppression of ocular neovascularization with siRNA targeting VEGF receptor 1. Gene Ther. 13:225-234.

21. Bitko, V., Musiyenko, A., Shulyayeva, O., and Barik, S. 2005. Inhibition of respiratory viruses by nasally administered siRNA. Nat. Med. 11:50-55.

22. Zhang, W., et al. 2005. Inhibition of respiratory syncytial virus infection with intranasal siRNA nanoparticles targeting the viral NS1 gene. Nat. Med. 11:56-62.

23. Reich, S.J., et al. 2003. Small interfering RNA (siRNA) targeting VEGF effectively inhibits ocular neovascularization in a mouse model. Mol. Vis. 9:210-216.

24. Dykxhoorn, D.M., and Lieberman, J. 2006. Running interference: prospects and obstacles to using small interfering RNAs as small molecule drugs. Annu. Rev. Biomed. Eng. 8:377-402.

25. Zimmermann, T.S., et al. 2006. RNAi-mediated gene silencing in non-human primates. Nature. 441:111-114.

26. Fedorov, Y., et al. 2005. Different delivery methodsdifferent expression profiles. Nat. Methods. 2:241.

27. Kumar, P., et al. 2007. Transvascular delivery of 
small interfering RNA to the central nervous system. Nature. 448:39-43.

28. Song, E., et al. 2005. Antibody mediated in vivo delivery of small interfering RNAs via cell-surface receptors. Nat. Biotechnol. 23:709-717.

29. McNamara, J.O., 2nd, et al. 2006. Cell type-specific delivery of siRNAs with aptamer-siRNA chimeras. Nat. Biotechnol. 24:1005-1015.

30. Wolfrum, C., et al. 2007. Mechanisms and optimization of in vivo delivery of lipophilic siRNAs. Nat. Biotechnol. 25:1149-1157.

31. Grimm, D., Pandey, K., and Kay, M.A. 2005. Adenoassociated virus vectors for short hairpin RNA expression. Methods Enzymol. 392:381-405.

32. Grimm, D., and Kay, M.A. 2003. From virus evolution to vector revolution: use of naturally occurring serotypes of adeno-associated virus (AAV) as novel vectors for human gene therapy. Curr. Gene Ther. 3:281-304.

33. Cavazzana-Calvo, M., and Fischer, A. 2007. Gene therapy for severe combined immunodeficiency: are we there yet? J. Clin. Invest. 117:1456-1465.

34. Herzog, R.W. 2007. Immune responses to AAV capsid: are mice not humans after all? Mol. Ther. 15:649-650.

35. Kay, M.A., et al. 2000. Evidence for gene transfer and expression of factor IX in haemophilia B patients treated with an AAV vector. Nat. Genet. 24:257-261.

36. Manno, C.S., et al. 2006. Successful transduction of liver in hemophilia by AAV-Factor IX and limitations imposed by the host immune response. Nat. Med. 12:342-347.

37. Marshall, E. 2000. Gene therapy on trial. Science. 288:951-957.

38. Grimm, D., et al. 2006. Fatality in mice due to oversaturation of cellular microRNA/short hairpin RNA pathways. Nature. 441:537-541.

39. McCarty, D.M., et al. 2003. Adeno-associated virus terminal repeat (TR) mutant generates self-complementary vectors to overcome the rate-limiting step to transduction in vivo. Gene Ther. 10:2112-2118.

40. McCarty, D.M., Monahan, P.E., and Samulski, R.J. 2001. Self-complementary recombinant adenoassociated virus (scAAV) vectors promote efficient transduction independently of DNA synthesis. Gene Ther. 8:1248-1254

41. Chen, C.C., et al. 2007. Long-term inhibition of hepatitis B virus in transgenic mice by doublestranded adeno-associated virus 8-delivered short hairpin RNA. Gene Ther. 14:11-19.

42. Paskowitz, D.M., et al. 2007. Rapid and stable knockdown of an endogenous gene in retinal pigment epithelium. Hum. Gene Ther. 18:871-880.

43. Xia, H., et al. 2004. RNAi suppresses polyglutamine-induced neurodegeneration in a model of spinocerebellar ataxia. Nat. Med. 10:816-820.

44. Boden, D., Pusch, O., Lee, F., Tucker, L., and Ramratnam, B. 2004. Efficient gene transfer of HIV-1specific short hairpin RNA into human lymphocytic cells using recombinant adeno-associated virus vectors. Mol. Ther. 9:396-402.

45. Zufferey, R., Dull, T., Mandel, R.J., Bukovsky, A., Quiroz, D., et al. 1998. Self-inactivating lentivirus vector for safe and efficient in vivo gene delivery. J. Virol. 72:9873-9880.

46. Chang, A.H., and Sadelain, M. 2007. The genetic engineering of hematopoietic stem cells: the rise of lentiviral vectors, the conundrum of the LTR, and the promise of lineage-restricted vectors. Mol. Ther. 15:445-456

47. Li, M., Li, H., and Rossi, J.J. 2006. RNAi in combination with a ribozyme and TAR decoy for treatment of HIV infection in hematopoietic cell gene therapy. Ann. N. Y. Acad. Sci. 1082:172-179.

48. Andersson, M.G., et al. 2005. Suppression of RNA interference by adenovirus virus-associated RNA. J. Virol. 79:9556-9565.

49. Yoo, J.Y., et al. 2007. VEGF-specific short hairpin
RNA-expressing oncolytic adenovirus elicits potent inhibition of angiogenesis and tumor growth. Mol. Ther. 15:295-302.

50. Wu, Z., Asokan, A., and Samulski, R.J. 2006. Adenoassociated virus serotypes: vector toolkit for human gene therapy. Mol. Ther. 14:316-327.

51. Sledz, C.A., Holko, M., de Veer, M.J., Silverman, R.H., and Williams, B.R. 2003. Activation of the interferon system by short-interfering RNAs. Nat. Cell Biol. 5:834-839.

52. Bridge, A.J., Pebernard, S., Ducraux, A., Nicoulaz, A.L., and Iggo, R. 2003. Induction of an interferon response by RNAi vectors in mammalian cells. Nat. Genet. 34:263-264.

53. Jackson, A.L., and Linsley, P.S. 2004. Noise amidst the silence: off-target effects of siRNAs? Trends Genet. 20:521-524.

54. Jackson, A.L., et al. 2003. Expression profiling reveals off-target gene regulation by RNAi. Nat. Biotechnol. 21:635-637.

55. Birmingham, A., et al. 2006. 3' UTR seed matches, but not overall identity, are associated with RNAi off-targets. Nat. Methods. 3:199-204.

56. Fedorov, Y., et al. 2006. Off-target effects by siRNA can induce toxic phenotype. RNA. 12:1188-1196.

57. Jackson, A.L., et al. 2006. Widespread siRNA "offtarget" transcript silencing mediated by seed region sequence complementarity. RNA. 12:1179-1187.

58. Lim, L.P., et al. 2005. Microarray analysis shows that some microRNAs downregulate large numbers of target mRNAs. Nature. 433:769-773.

59. Lin, X., et al. 2005. siRNA-mediated off-target gene silencing triggered by a $7 \mathrm{nt}$ complementation. Nucleic Acids Res. 33:4527-4535.

60 . Hornung, V., et al. 2005. Sequence-specific potent induction of IFN-alpha by short interfering RNA in plasmacytoid dendritic cells through TLR7. Nat. Med. 11:263-270.

61. Judge, A.D., Bola, G., Lee, A.C., and MacLachlan, I 2006. Design of noninflammatory synthetic siRNA mediating potent gene silencing in vivo. Mol. Ther 13:494-505.

62. Judge, A.D., et al. 2005. Sequence-dependent stimulation of the mammalian innate immune response by synthetic siRNA. Nat. Biotechnol. 23:457-462.

63. Morrissey, D.V., et al. 2005. Potent and persistent in vivo anti-HBV activity of chemically modified siRNAs. Nat. Biotechnol. 23:1002-1007.

64. Yi, R., Doehle, B.P., Qin, Y., Macara, I.G., and Cullen, B.R. 2005. Overexpression of exportin 5 enhances RNA interference mediated by short hairpin RNAs and microRNAs. RNA. 11:220-226.

65. Yi, R., Qin, Y., Macara, I.G., and Cullen, B.R. 2003. Exportin-5 mediates the nuclear export of premicroRNAs and short hairpin RNAs. Genes Dev. 17:3011-3016

66. John, M., et al. 2007. Effective RNAi-mediated gene silencing without interruption of the endogenous microRNA pathway. Nature. 449:745-747.

67. Nishitsuji, H., Kohara, M., Kannagi, M., and Masuda, T. 2006. Effective suppression of human immunodeficiency virus type 1 through a combination of short- or long-hairpin RNAs targeting essential sequences for retroviral integration. J. Virol. 80:7658-7666.

68. Hemmings-Mieszczak, M., et al. 2003. Independent combinatorial effect of antisense oligonucleotides and RNAi-mediated specific inhibition of the recombinant rat $\mathrm{P} 2 \mathrm{X} 3$ receptor. Nucleic Acids Res. 31:2117-2126

69. Hutvagner, G., Simard, M.J., Mello, C.C., and Zamore, P.D. 2004. Sequence-specific inhibition of small RNA function. PLoS Biol. 2:E98.

70. Sasaki, T., Shiohama, A., Minoshima, S., and Shimizu, N. 2003. Identification of eight members of the Argonaute family in the human genome small star, filled. Genomics. 82:323-330.
71. Wiznerowicz, M., Szulc, J., and Trono, D. 2006. Tuning silence: conditional systems for RNA interference. Nat. Methods. 3:682-688.

72. Schwarz, D.S., et al. 2006. Designing siRNA that distinguish between genes that differ by a single nucleotide. PLoS Genet. 2:e140.

73. Sumimoto, H., et al. 2006. Effective inhibition of cell growth and invasion of melanoma by combined suppression of BRAF (V599E) and Skp2 with lentiviral RNAi. Int. J. Cancer. 118:472-476.

74. Pichler, A., Zelcer, N., Prior, J.L., Kuil, A.J., and Piwnica-Worms, D. 2005. In vivo RNA interferencemediated ablation of MDR1 P-glycoprotein. Clin. Cancer Res. 11:4487-4494.

75. Zhang, X., et al. 2004. Enhancement of hypoxiainduced tumor cell death in vitro and radiation therapy in vivo by use of small interfering RNA targeted to hypoxia-inducible factor-1alpha. Cancer Res. 64:8139-8142.

76. Samakoglu, S., et al. 2006. A genetic strategy to treat sickle cell anemia by coregulating globin transgene expression and RNA interference. Nat. Biotechnol. 24:89-94.

77. Patel, D.J., et al. 2006. Structural biology of RNA silencing and its functional implications. Cold Spring Harb. Symp. Quant. Biol. 71:81-93.

78. Bagga, S., et al. 2005. Regulation by let-7 and lin-4 miRNAs results in target mRNA degradation. Cell. 122:553-563.

79. Tang, G. 2005. siRNA and miRNA: an insight into RISCs. Trends Biochem. Sci. 30:106-114.

80. Lewis, B.P., Burge, C.B., and Bartel, D.P. 2005. Conserved seed pairing, often flanked by adenosines, indicates that thousands of human genes are microRNA targets. Cell. 120:15-20.

81. Lewis, B.P., Shih, I.H., Jones-Rhoades, M.W., Bartel, D.P., and Burge, C.B. 2003. Prediction of mammalian microRNA targets. Cell. 115:787-798.

82. Hammond, S.M. 2006. MicroRNA therapeutics: a new niche for antisense nucleic acids. Trends Mol. Med. 12:99-101.

83. Ebert, M.S., Neilson, J.R., and Sharp, P.A. 2007. MicroRNA sponges: competitive inhibitors of small RNAs in mammalian cells. Nat. Methods. 4:721-726.

84. Krutzfeldt, J., et al. 2005. Silencing of microRNAs in vivo with 'antagomirs'. Nature. 438:685-689.

85. Orom, U.A., Kauppinen, S., and Lund, A.H. 2006. LNA-modified oligonucleotides mediate specific inhibition of microRNA function. Gene. 372:137-141.

86. Lu, J., et al. 2005. MicroRNA expression profiles classify human cancers. Nature. 435:834-838.

87. Jopling, C.L., Yi, M., Lancaster, A.M., Lemon, S.M., and Sarnow, P. 2005. Modulation of hepatitis C virus RNA abundance by a liver-specific MicroRNA. Science. 309:1577-1581.

88. Brown, B.D., Venneri, M.A., Zingale, A., Sergi Sergi, L., and Naldini, L. 2006. Endogenous microRNA regulation suppresses transgene expression in hematopoietic lineages and enables stable gene transfer. Nat. Med. 12:585-591.

89. Brown, B.D., et al. 2007. A microRNA-regulated lentiviral vector mediates stable correction of hemophilia B mice. Blood. doi:10.1182/blood-2007-03-078493.

90. Morris, K.V., Chan, S.W., Jacobsen, S.E., and Looney, D.J. 2004. Small interfering RNA-induced transcriptional gene silencing in human cells. Science. 305:1289-1292.

91. Kim, D.H., Villeneuve, L.M., Morris, K.V., and Rossi, J.J. 2006. Argonaute-1 directs siRNA-mediated transcriptional gene silencing in human cells. Nat. Struct. Mol. Biol. 13:793-797.

92. Janowski, B.A., et al. 2006. Involvement of AGO1 and $\mathrm{AGO} 2$ in mammalian transcriptional silencing. Nat. Struct. Mol. Biol. 13:787-792.

93. Bernards, R. 2006. Exploring the uses of RNAi - gene knockdown and the Nobel Prize. N. Engl. J. Med. 355:2391-2393. 